\title{
Review Article: Systemic Lupus Erythematosus
}

\author{
Habib $\mathrm{N}^{1 *}$, Rashid $\mathrm{M}^{2}$, Afzal $\mathrm{F}^{3}$, Afrose $\mathrm{T}^{4}$ and Nangarath EKC ${ }^{1}$ \\ ${ }^{1}$ Department of Physiology, Quest International University, Malaysia \\ ${ }^{2}$ Department of Medicine, Quest International University, Malaysia \\ ${ }^{3}$ Department of Oculoplasty, Ispahani Islamia Eye Institute and Hospital, Bangladesh \\ ${ }^{4}$ Department of Community, Medicine AIMST University FOM; AIMST University, Malaysia
}

\section{Review Article \\ Volume 5 Issue 1}

Received Date: January 02, 2020

Published Date: January 24, 2020

DOI: $10.23880 /$ oajmb-16000158

*Corresponding author: Nasrin Habib, Senior Lecturer, Department of Physiology, Quest

International University, Perak Malaysia, Email: nasrin.habib@qiup.edu.my

\section{Abstract}

Systemic lupus erythematosus or SLE is a persistent heterogeneous autoimmune disease that affects multisystem of the body. It is distinguished by acute and chronic inflammation of various tissues and even organs of the body principally the skin and joints. Systemic lupus erythematosus is a multisystem disorder and hence, it can affect any tissues, organs and even systems of the body. There are few categories of lupus for instance, lupus dermatitis or cutaneous lupus erythematosus (CLE) that affects the skin and causes malar rash, discoid lupus erythematosus (DLE) as well as systemic lupus erythematosus that causes damage to single or multiple internal organs. The damage is due to the inflammation that is caused by direct antibody reaction to the body tissues as well the deposition of immune complexes. Glucocorticoids, immunosuppressant, and antimalarial are the combination therapy used to treat SLE besides providing counseling and awareness. Lupus erythematosus in any form particularly systemic lupus erythematosus (SLE) are prevalent in women compared to men with ratio of 6:1. It has the tendency to affect all ages but most frequently attacks women of aged 20 to 45 years old compared to men. On the other hand, if lupus erythematosus causes damage to internal organs either single or multiple, it is known as systemic lupus erythematosus. The damage is due to the inflammation that is caused by direct antibody reaction to the body tissues as well the deposition of immune complexes.

Keywords: SLE; Lupus Erythematosus; Autoimmune Disorder

Abbreviations: SLE: Systemic Lupus Erythematosus; CLE: Cutaneous Lupus Erythematosus; DLE: Discoid Lupus Erythematosus; APC: Antigen Presenting Cells; TLR: TollLike Receptor; MHC: Major Histocompatibility Complex; HLA: Human Leukocyte Antigen; AD: Autoimmune Disorder.

\section{Introduction}

Systemic lupus erythematosus or SLE is a persistent heterogeneous autoimmune disease that affects multisystem of the body. Autoimmune disorder by definition is disease manifesting from the immune response against self-antigens.
The immune system is a network of cells, tissues, and organs that work together with soluble humoral components (production of antibody against an infection) for instance, antibodies or complement to protect the body against attacks by foreign invaders. Immune system of the body has the ability to distinguish between self and foreign (microbial) agents. If these mechanisms fail in the case of autoimmune disorders, the immune system will attack the individual's own cells and tissues by production of autoantibodies with protean clinical manifestation. In the case of systemic lupus erythematosus, the body is unable to produce normal antibodies against any infection. Instead, the body will start 


\section{Open Access Journal of Microbiology \& Biotechnology}

producing abnormal antibodies that is unable to recognize self and foreign microbial agents. These abnormal antibodies recognize self-antigens as foreign and consequently, it will bind and attack the self-antigens rather than foreign infectious agents. These abnormal antibodies are known as autoantibodies [1]. These autoantibodies are directed against intracellular targets. The typical types of autoantibodies that are present in at least 95\% of individuals with SLE are antinuclear antibodies (ANAs). Others for instance, Antidouble-stranded DNA (dsDNA), anti-smith (anti-Sm), antiRo, and anti-La antibodies are rare [2]. The heterogeneity systemic lupus erythematosus is a multisystem disorder and hence, it can affect any tissues, organs and even systems of the body. For instance, when it affects the skin and causes butterfly rash, it is known as lupus dermatitis or cutaneous lupus erythematosus (CLE). Discoid lupus erythematosus (DLE) is a form of cutaneous lupus erythematosus [3].

\section{Etiology}

Specific genetic mutation such as inborn deficit of C1q, C2, and C4, as well as several polymorphisms for instance, interferon regulatory factor 5 and protein tyrosine phosphatase N22 and familial clustering of SLE or other autoimmune disease have contributed to genetic susceptibility to SLE. In addition, certain human leukocyte antigen (HLA) types including HLA-B8, HLA-DR2, and HLADR3 most commonly present in patients suffering from SLE [4]. Also, defect in complement tends to have the highest risk of SLE disease, and mutations in TREX1 point to impaired regulation of endogenous nucleic acids as well contributes to the genetic predisposition to SLE [5]. Moreover, multiple genetic linkages including 1q23, 2q3537, 6p2111, and 12q24 suggest close relation with SLE [6]. Environmental exposures such as ultraviolet light exposure and certain viral infections for instance, Epstein-Barr virus may partake a role in susceptible individuals [7].

\section{Pathogenesis}

The definite pathogenesis of systemic lupus erythematosus still remains vague. Genetic triggers, environmental triggers, autoantibodies as well as immune complexes and complement have been identified to cause the interference in innate and adaptive immune regulatory mechanism. Intercession in the immune regulatory mechanism will further results in the interruption in apoptotic cell clearance, cytokines, B-cell immunity, and T-cell signaling that will progress in SLE pathogenesis. In general, the apoptotic cells will initiate signals like cellsurface protein such as phophatidylserine that will trigger the immune cells as well as macrophages and dendritic cells to phagocyte the excessive apoptotic debris. SLE is strongly associated with defects in the apoptotic clearance. Inability to remove the apoptotic remnants effectively causes the antigen presenting cells (APC) to arrest the fragments of the nuclear particles that will further cause them to interface with T \& B cells. Impairment of immune regulatory mechanism further leads to increase in antigenic load and overproduction and overactive of $\mathrm{T}$ cells as well as the defect in suppression of $B$ cell activation by the immune cells [8]. Further interaction will eventually leads to the development of the antinuclear antibodies which is the hallmark of this disease. Production of autoantibodies will release the immune complexes that will lead to inflammation, tissue injury and cell and organ damage that will progress in SLE disease. Genetic factors for instance, polymorphisms in components of the toll-like receptor (TLR) that usually increases the production of type 1 interferon as well as interferon regulatory factor 5 and protein tyrosine phosphatase N22 and familial clustering of SLE have been identified to contribute to the genetic susceptibility to SLE. In addition, major histocompatibility complex (MHC) class II alleles with human leukocyte antigen (HLA) types including HLA-B8, HLA-DR2, and HLA-DR3 are most commonly associated with SLE [5].

\section{Statistics of Systemic Lupus Erythematosus in Malaysia}

Lupus erythematosus in any form particularly systemic lupus erythematosus (SLE) are prevalent in women compared to men with ratio of $6: 1$ [9]. About $90 \%$ cases reported out of SLE are women while the other $10 \%$ are men and children. It has the tendency to affect all ages but most frequently it attacks women of aged 20 to 45 years old compared to men. In Malaysia, it has been estimated that more than 10,000 people have been diagnosed from SLE over the past 30 years but this count is only at the tip of the iceberg [10]. There are many people who are suffering from SLE that have not been diagnosed according to the Malaysian SLE Association as they did not approach the doctor for consultation.

\section{Signs and symptoms}

In general, patients who suffer from systemic lupus erythematosus usually come over with complaints of general symptoms such as fatigue, weight loss, fever as well as myalgia and arthralgia [11]. Fatigue is the commonest symptom that patients often complain about that can actually make them enfeeble. The hallmark symptom of this disease is red, butterfly rash on the skin particularly on the cheeks. In terms of dermatology, there are three types of lesions which are Acute Cutaneous Lupus, Sub-Acute Cutaneous Lupus and Chronic Cutaneous Lupus. Cases have been reported that up to $61.3 \%$ of SLE patients suffer from red, butterfly rash on the cheeks sparing the nasolabial folds and the degree of intensity may vary [12]. Also, red plaques without scarring may develop on the skin area that is exposed to the Sun and 


\section{Open Access Journal of Microbiology \& Biotechnology}

it is known as acute cutaneous lupus. Non-scarring and soft skin lesions that are often intermittent are known as subacute cutaneous lupus. On the other hand, in discoid lesions or chronic cutaneous lupus, red plaques may be accompanied by alopecia which is loss of hair at particular area where the red plaques are developed [9]. Besides, oral and nasal ulcers may also manifest in 51\% of SLE patients and usually are quite painful [12]. Besides, musculoskeletal system is the commonest system that affects most of the patients who are diagnosed from systemic lupus erythematosus. The typical symptoms that patients usually present with are arthritis and arthralgia that usually affects the small joints of the hands, wrists as well as the knees [3]. In some cases, patients have come up with symptoms of dysphagia, heartburn, abdominal pain, as well as vasculitis. Clinical pericarditis and pleuritis have been reported up to $63 \%$ of the autopsy cases $[1,13,14]$.

\section{Diagnosis}

According to 2012 SLICC Criteria, an individual is being diagnosed suffering from SLE if the biopsy revealed nephritis with ANA or antidsDNA antibodies or he/she fulfill four of the clinical criteria with at least one clinical criteria and at least one immunological criteria. Clinical criteria includes acute or chronic cutaneous lupus, oral or nasal ulcers, nonscarring alopecia, synovitis, serositis with pericardial more than 24 hours, renal of which $>500 \mathrm{mg}$ proteinuria/24 hours or RBC casts, neurologic symptoms which includes seizures, psychosis, mononeuritis multiplex, myelitis, peripheral or cranial neuropathy or acute confusional state, hemolytic anemia, lymphopenia or thrombocytopenia [6]. In terms of immunological criteria, individual who are diagnosed to be suffering from SLE shows at least one positive result for ANA or antidsDNA or antiSmith or antiphospholipid antibodies or Direct Coombs test in the absence of hemolytic anemia [3]. Direct immunofluorescence also being carried out and the typical finding includes antibody deposition which are typically granular and composed of IgG or IgM or sometimes might be IgA as well at the dermal-epidermal junction and around hair follicles [9].

\section{Treatment}

To treat SLE completely is impossible and onerous as it causes relapse. Thus, the ultimate goal of SLE treatment is to stop the organ inflammation, avert the irreversible organ damage as well as to suppress the immune response causing the inflammation and flares and to lower down the intensity of pain. The drugs that are used to treat SLE are glucocorticoids and the target dose of glucocorticoids should be $0.25 \mathrm{mg} /$ $\mathrm{kg}$ every other day for 2 to 3 months [15]. Patients who are under chronic steroid therapy should be evaluated closely as they are prone for fever or any infection. Next, antimalarial drugs such as hydroxychloroquine and chloroquine are prescribed for skin and mucocutaneous manifestations [16]. Study has been done and proven that the use of antimalarial drugs has resulted in greater than $50 \%$ reduction of SLE manifestation. Immunosuppressive drugs like azathioprine, methotrexate or cyclophosphamide are given to the patients who are suffering from severe renal or cerebral disease at which the other therapies have failed or for those who are unable to tolerate with corticosteroids [17].

\section{Discussion}

The outcome of this study reveals the latest usage of rituximab as the drug of choice in the treatment of SLE. In the randomized controlled trials, it has shown some improvement in the prognosis of SLE but the usage of Rituximab reveals negative results in terms of renal and non-renal [6]. The clinical research of this drug has not been thoroughly tested yet and further study and trials need to be done to improvise so there will be less adverse reaction and better prognosis as the outcome for renal was negative. If this drug is possible to treat the patients in terms of renal, it would be a great discovery to safe most of the SLE patients who ended up of having end stage kidney disease. On the other hand, in March 2011, the US Food and Drug Administration have given the green light for the usage of Belimumab as a part of the treatment of SLE in the adults [9]. It has good prognosis in terms of reducing the time to develop flares and lower the exposure towards glucocorticoids. Based on the latest study, we found that the five year survival rate has improved in more than $90 \%$ of SLE patients with the usage of immunosuppressant therapy [6]. However, most of the patients develop relapse and their condition deteriorate and this worsen in SLE patients with lupus nephritis [18-20].

\section{Conclusion}

The mortality rate of patients who suffer from SLE is thrice higher than the general populations. Studies and results obtained highlight the desideratum for further research and clinical progress. More research needs to be done together with clinical trials to improve the prognosis and the quality of life of patients who suffers from SLE. Vigilant follow up, inventing latest most effective drugs as well as improving the diagnostic research methods will one day prove that SLE can be treated with complete convalescence.

\section{References}

1. Aranow C, Diamond B, Mackay M (2013) Systemic Lupus Erythematosus. Part Six: Systemic Immune Diseases pp: 603-620.

2. Julia FS, Karen HC (2015) Epidemiology and Classification of Systemic Lupus Erythematosus. Rheumatology 124: 1021-1025. 


\section{Open Access Journal of Microbiology \& Biotechnology}

3. Abelson A, Bharadwaj S, Wright B (2010) Systemic Lupus Erythematosus. Section 13 Rheumatology and Immunology pp: 1169-1176.

4. Rebecca ES, Schanberg LE, Stacy PA (2016) Systemic Lupus Erythematosus. Nelson Textbook of Pediatrics Chapter 158: 1176-1181.

5. Mary KC (2013) Etiology and Pathogenesis of Systemic Lupus Erythematosus. Systemic Lupus Erythematosus and Related Syndrome pp: 1269-1282.

6. Ferri FF (2016) Systemic Lupus Erythematosus. Ferri's Clinical Advisor.

7. Yazdany J (2013) Systemic Lupus Erythematosus.

8. Mok CC, Lau CS (2003) Pathogenesis of Systemic Lupus Erythematosus. J Clin Pathol Chapter 56(7): 481-490.

9. Lee LA, Werth VP (2012) Lupus Erythematosus. Dermatology 41: 615-629.

10. Malaysian SLE Association (2016) Statistics of Systemic Lupus Erythematosus in Malaysia.

11. Robert GL (2011) The Clinical Presentation of Systemic Lupus Erythematosus. $5^{\text {th }}$ (Edn.), pp: 525-539.

12. Jasmin R, Sockalingam S, Cheah TE, Goh KJ (2013)
Systemic Lupus Erythematosus in The Multiethnic Malaysian Population: Disease Expression and Ethnic Differences Revisited. Lupus 22(9): 967-971.

13. Kao AH, Susan M (2016) Systemic Lupus Erythematosus. Andreoli and Carpenter's Cecil Essentials of Medicine $9^{\text {th }}$ (Edn.), pp: 780-786.

14. Muscal E, Brey RL (2010) Neurological Manifestations of Systemic Lupus Erythematosus in Children and Adults. Neurol Clin 28(1): 61-73.

15. Jessica JM, Rahman A (2006) Systemic Lupus Erythematosus. Orphanet Journal of Rare Diseases.

16. George B, Antonios F, Boumpas DT (2013) Treatment of Systemic Lupus Erythematosus. Kelley's Textbook of Rheumatology 81: 1304-1330.

17. Susanne MB, Silverman ED (2005) Systemic Lupus Erythematosus. Pediatr Clin North Am 52(2): 443-467.

18. James GA (2013) Systemic Lupus Erythematosus.

19. Larissa L, Murphy G, David I (2014) Systemic Lupus Erythematosus. Lancet 384: 1878-1888.

20. Mary KC (2016) Systemic Lupus Erythematosus. Systemic Lupus Erythematosus Chapter 266: 17691777. 